\title{
Substance Use Disorder and comorbid adult ADHD. The importance of an early diagnosis. A case report
}

\author{
A. Rodríguez Sotelo', I. Carrera Machado². \\ ${ }^{1}$ Complexo Hospitalario Universitario A Coruña, Spain. ${ }^{2}$ ACLAD, A Coruña, Spain
}

\section{INTRODUCTION}

Attention Deficit Hyperactivity Disorder (ADHD) is a neurodevelopmental disorder that was originally defined in children but is now recognized to persist into adulthood, thus affecting up to a $57 \%$ of patients with a history of childhood ${ }^{1}$

Furthermore, there is a strong relation between ADHD and Substance Use Disorders (SUD), with an estimated prevalence of about $23 \%{ }^{2}$. The presence of this Dual Pathology is associated with a poorer course and a less favorable prognosis of drug abuse ${ }^{3}$.

Nevertheless, ADHD remains under-diagnosed in addict patients, possibly due to the frequent overlap of symptoms. often accompanied by other psychiatric comorbidities. These comorbid disorders may indeed lead the patients to seek psychiatric care, but since their ADHD symptoms are often mistaken for those of their comorbidities, patients are unlikely to receive a proper ADHD diagnosis; thus resulting in a poor outcome and long-term debilitating effects on quality of life.

\section{OBJECTIVES}

Our aim is to highlight the importance of an early diagnosis of comorbid ADHD in adult patients with SUD, by presenting a case report of a female patient with Dual Pathology who received a diagnosis of ADHD at the age of 44, although she had developed relevant ADHD symptoms since childhood which were misdiagnosed over the years of Psychiatric follow-up.

\section{METHODS}

Pilar was a 44 year old woman, single, with no children. No familiy history of mental health problems.

Her first contact with Mental Health system was in her childhood due to behavioural disturbances. She was described as an impulsive, restless and distracted girl.

At the young age of 12, she iniciated alcohol and cannabis use and later on, at the age of 14 she was also consuming amphetamines, cocaine and heroine.

She took contact with an Addiction Unit at the age of 24, and was diagnosed of Opioids Dependence (304.00) and abuse to multiple substances (Alcohol, Cannabis, Cocaine, Amphetamines). She was included in a methadone maintenance treatment (MMT)(in which she still remains) and was also treated with different antidepressants over the years for referred low mood. As medical comorbodities, she was positive for HIV and HCV infection.

Clinical outcomes throughout these years were poor, she had multiple dropouts for psychiatric and antiviral treatments and substance relapses were frequent. She also had a significant personal and social impairment that led her to a chronic low mood and self-steem.

It was not until several years of follow-up, when the patient's clinical course was carefully reevaluated, and so ADHD symptoms arose. Screening tests were carried out and supported the clinical suspicion of a comorbid ADHD.

She was initially treated withl long-acting methylphenidate (Concerta $\AA$ ) that the patient abandoned due to gastrointestinal side effects, and thus a second trial was made with lisdexanphetamine (Elvanse®).

After several months of treatment, a global improvent was observed. The patient has remained abstinent since, except for cannabis use and has accomplished a long-term stable medical follow-up. This improvent was corroborated in CAARS Scale (figure 1).

\section{DISCUSSION}

While ADHD is a common, identifiable and treatable disorder, it remains underdiagnosed in adult population.

The major impact of ADHD on patients' well-being and its negative impact on their social, family and working lives, along with its strong association with Substance Use Disorder, forces us to claim pshychiatrists' attention to this disorder and increase awareness about adult manifestations of ADHD.

Clinical psychiatrists should always assess retrospectively any relevant psychiatric symptoms in childhood, even in the absence of a prior ADHD diagnosis.

Finally, since adults with ADHD are more likely to seek psychiatric healthcare for treatment of their comorbid disorders (specially substance use disorders) rather than for ADHD, similarly to the patient of our case report, their symptoms can be mistaken for those of their comorbidities and thus masking the underlying ADHD
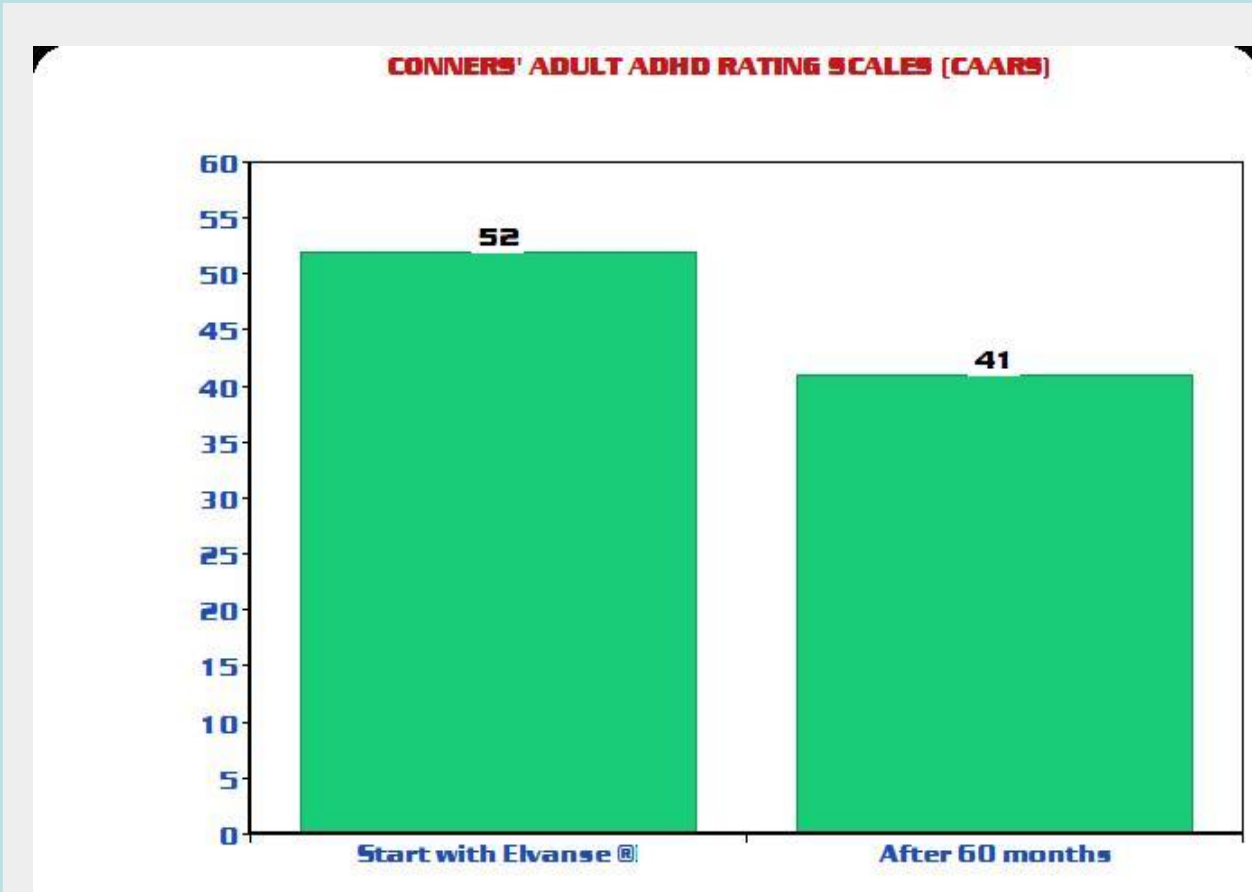

FIGURE 1. Clinical response after 60 months of treatment with Lisdexamfetamine

\section{CONCLUSIONS}

Early diagnose of ADHD in addict patients may lead to better clinical outcomes, not only of the proper disorder, but also for the comorbid SUD.

Psychiatrists should be aware of this pathology, which we think remains underdiagnosed in clinical practice.

\section{REFERENCES}

${ }^{1}$ Fayyad J, Sampson NA, Hwang I, et al; WHO World Mental Health Survey Collaborators. The descriptive epidemiology of DSM-IV Adult ADHD in the World Health Organization World Mental Health 